\title{
Do optical quarks exist in the free space? A scalar treatment.
}

\author{
Volyar Alexander V. \\ General Physics Department, Taurida National University, Simferopol, Ukraine, \\ e-mail: volyar@crimea.edu
}

Received: 13.12 .2012

\begin{abstract}
We have considered a new type of singular beams called as optical quarks. They have fractional topological charges being equal to half an integer and they possess rather unique properties. There are four types of optical quarks, even and odd ones, which reveal the opposite signs of topological charges. The sums or differences of the even and odd quarks form standard vortex or non-vortex beams with the topological charges of integer order. All the quarks in the same beam annihilate and the beam vanishes. The analysis of angular spectra of the optical quarks shows that the latter represent structurally unstable forms of field under condition of free-space propagation. We have analysed their propagation properties for different types of beam envelope, including a symmetric beam array with discrete optical quarks. We have discussed the properties of possible structurally stable forms of the quarks and the media capable of maintaining the optical quarks.
\end{abstract}

Keywords: optical quarks, fractional topological charge, erf-G beams

PACS: $42.50 . \mathrm{Tx}$

UDC: 535.1

\section{Introduction}

As far back as in the beginning of 1990s, Soskin et al. [1, 2] have wondered at a 'strange' behaviour of the simplest singular beams with fractional topological charges. It turns out that the inherent property of such beams is that the initial field distribution is not recovered during propagation along any beam length, while the optical vortex with a fractional topological charge is not nucleated at any beam cross section. Indeed, a broken axial symmetry of the beam does not permit reconstructing the propagating field. The immediate inference is that the vortex beams with the fractional topological charges cannot exist in principle. Although such a simple statement does not need a strong confirmation at all, the work by Berry [3] has ignited a heated discussion. Berry has considered the process of diffraction for a Gaussian $(G)$ beam by a spiral phase plate with the fractional phase step. The evolution of the diffracted beam manifests itself in the form of beam fracture, with chains of singly charged optical vortices. However, the major point has been that the beam could carry over a fractional orbital angular momentum (OAM).

An avalanche of subsequent studies has surpassed all imagination (see, e.g., [4-10] and references therein). The detailed analysis has shown that the fractional optical vortex splits into an infinite series of integer-order vortices, while the OAM of the beam is defined by contributions of integer-order optical vortices. Although it seems at the first sight that fractionalising the OAM of the beam contradicts the foundations of quantum mechanics, the authors of the work [11] have shown the mixed stated of photons to be able to carry over the fractional OAMs. At the same time, according to the results [3], the fractional-vortex beam must inevitably be destroyed while propagating, because of different phase velocities of partial elementary beams involved. Nevertheless, 
the recent studies $[12,13]$ have demonstrated availability of spatially invariant beams with fractional OAMs and, in particular, with fractional optical vortices [12] (so-called erf-G beams).

It is important to stress that we also come upon a pattern of fractional edge dislocations in the Ahoronov-Bohm effect and in the case of surface waves occurring in a swimming pool above a sink [14]. Such unusual properties of the fractional-vortex beams compel to peer more attentively into the structure of spatially invariant fractional-vortex beams. The aim of the present article is to analyse the structural features of elementary fractional-vortex beams in the free space.

\section{2. erf-G beams as anticipation of optical quarks}

\subsection{Fundamental quark properties}

We have shown in the study [12] that the error function-Gaussian beams (erf-G beams) bearing optical vortices with the topological charges $l= \pm 1 / 2$ belong to the strong solutions of the vector paraxial wave equation and refer to a set of so-called standard paraxial beams (Hermite-Gaussian (HG), Laguerre-Gaussian (LG), Bessel-Gaussian (BG), etc.), with a complex argument. In contrast to the usual standard beams (e.g., HG, Laguerre (L), G or BG ones), the erf-G beams have a non-factorising form, i.e. their azimuthal $(\varphi)$ and radial $(r)$ variables are not separated. The scalar erf-G beam may be written in the form

$$
\Psi_{s}=-\frac{2 i \sqrt{\pi} e^{i s \varphi / 2}}{\mathfrak{R}} N G\left\{e^{-\mathfrak{R}^{2} / 2} \operatorname{erf}\left(i \Re \sin \frac{\varphi}{2}\right)+s e^{\mathfrak{R}^{2} / 2} \operatorname{erf}\left(\mathfrak{R} \cos \frac{\varphi}{2}\right)\right\},
$$

where $\operatorname{erf}(x)$ stands for the error function, $\mathfrak{R}=\sqrt{2 \frac{K r}{\sigma}}, \quad G=\exp \left(-\frac{r^{2}}{w_{0}^{2} \sigma}\right) / \sigma$, $N=\exp \left(-\frac{K^{2} w_{0}^{2}}{4 \sigma}\right), \sigma=1-i z / z_{0}$, and $z_{0}=k w_{0}^{2} / 2$. Here $w_{0}$ is the radius of the beam waist at $z=0, k$ the wavenumber, $s= \pm 1$, and the free parameter $K$ can acquire arbitrary values, including complex ones. The field distribution given by Eq. (1) depends on the free parameter $K$.

The intensity and phase distributions shown in Fig. 1 illustrate variations of beam structure for both the real and imaginary $K$ parameters at the initial plane $z=0$. In the case of purely imaginary $K$ parameter, the field represents a complex set of singly charged optical vortices, which evolve in a complex manner when propagating. When the $K$ parameter is purely real, the intensity distribution acquires a $C$-like shape. The ray along $\varphi=0$ is something like a broken edge dislocation that matches two edges of the wave function given by Eq. (1), with different phases in a one-half optical vortex. Even very small shifts from the initial plane break a spiral-like phase structure in Fig. 1d, resulting in phase smoothing [12].

Near the beam axis where $K r$ is very small ( $K r<<1$ ), the wave function of the erf-G beam given by Eq. (1) may be presented as

$$
\Psi_{s} \approx 2 \sqrt{\pi} e^{i s \varphi / 2} N G\left\{e^{-\Re^{2} / 2} \sin \frac{\varphi}{2}-i s e^{\mathfrak{R}^{2} / 2} \cos \frac{\varphi}{2}\right\} .
$$

Notice that the two terms in Eq. (2) have much to do with the forms of the fractional-vortex beams suggested by Soskin et al. in the work [1]. Let us write out a generalised form of such wave constructions at the initial plane $z=0$ and outline their basic properties: 


$$
\begin{aligned}
& Q_{e v}^{+, m}=\cos \left(m \frac{\varphi}{2}\right) e^{i m \frac{\varphi}{2}} F(r), \\
& Q_{o d}^{+, m}=-i \sin \left(m \frac{\varphi}{2}\right) e^{i m \frac{\varphi}{2}} F(r), \\
& Q_{e v}^{-, m}=-\cos \left(m \frac{\varphi}{2}\right) e^{-i m \frac{\varphi}{2}} F(r), \\
& Q_{o d}^{-, m}=-i \sin \left(m \frac{\varphi}{2}\right) e^{-i m \frac{\varphi}{2}} F(r),
\end{aligned}
$$

where $F(r)$ is the radial envelope of the standard paraxial beam and $m=2 m^{\prime}+1$ is an odd number.

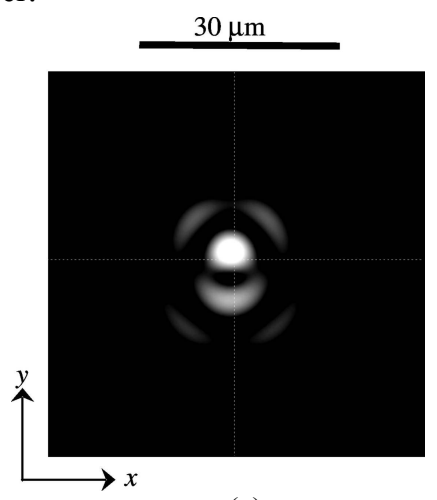

(a)

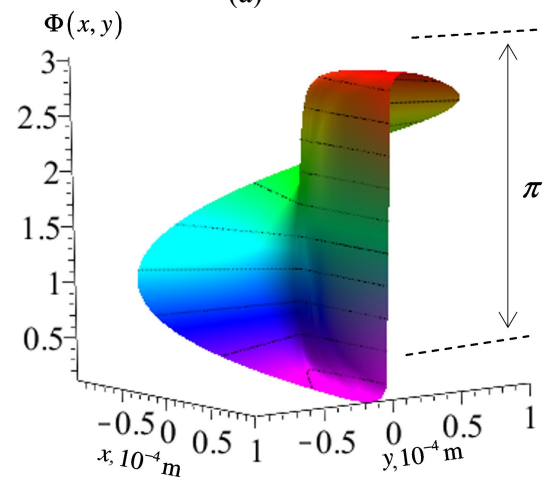

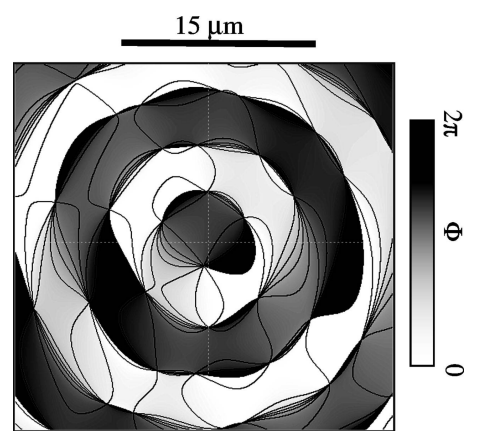

(b)

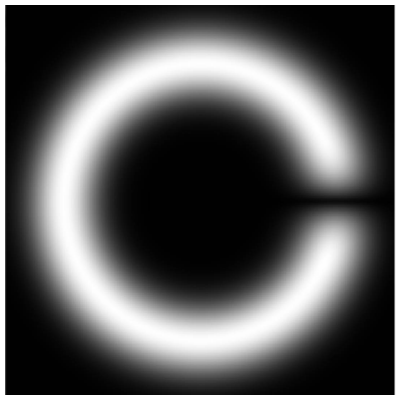

(c)

Fig. 1. Intensity (a, c) and phase (b, d) distributions for the erf-G beam with $w_{0}=35 \mu \mathrm{m}$ in the initial plane $z=0:(\mathrm{a}, \mathrm{b}) K_{\perp}=i 6 \times 10^{5} \mathrm{~m}^{-1}$ and $(\mathrm{c}, \mathrm{d})$ (d)

An arbitrary non-vortex beam can be presented as a superposition of the wave elements given by Eqs. (3)-(6):

$$
F(r)=Q_{e v}^{+, m}+Q_{o d}^{+, m} \text { or } F(r)=-\left(Q_{e v}^{-, m}+Q_{o d}^{-, m}\right) .
$$

Quite similar, one can define an arbitrary paraxial vortex beam with the odd topological charge:

$$
F(r) e^{i m \varphi}=Q_{e v}^{+, m}-Q_{o d}^{+, m} \text { and } F(r) e^{-i m \varphi}=Q_{o d}^{-, m}-Q_{e v}^{-, m} .
$$

Correspondingly, the beams with the edge dislocations may be written as

$$
F(r) \cos (m \varphi)=\left\{Q_{e v}^{+, m}-Q_{o d}^{+, m}+Q_{o d}^{-, m}-Q_{e v}^{-, m}\right\} / 2,
$$




$$
F(r) \sin (m \varphi)=\left\{Q_{e v}^{+, m}-Q_{o d}^{+, m}-Q_{o d}^{-, m}+Q_{e v}^{-, m}\right\} / 2 i .
$$

However, since the vortex beams of high orders are unstable with respect to slight perturbations, later on we will focus our attention only on the simplest vortex beams with $m= \pm 1$.

Another basic property is that the sum all the wave constructions given by Eqs. (3)-(6) vanishes:

$$
Q_{e v}^{+, m}+Q_{o d}^{+, m}+Q_{e v}^{-, m}+Q_{o d}^{-, m}=0 .
$$

In analogy with the Gell-Mann quark model of hadrons [15], we will call such wave constructions as optical quarks. In this way the wave constructions $Q_{o d}^{-, m}$ and $Q_{e v}^{-, m}$ may be treated as antiquarks. Eqs. (7) and (8) can be read such that a superposition of two even and odd quarks or antiquarks forms a non-vortex beam, while their difference represents a vortex-beam. At the same time, the occurrence of all the quarks and anti-quarks results in their total annihilation (see Eq. (11)).
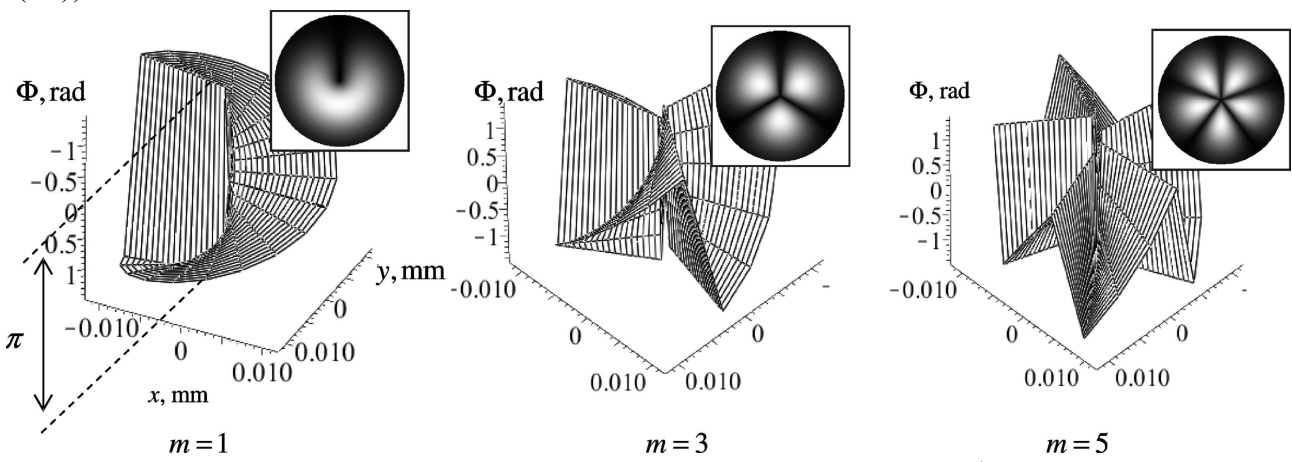

Fig. 2. Intensity $(I)$ and phase $(\Phi)$ distributions for the optical quark $Q_{e v}^{+, 1}$ with a standard envelope $F(r)=\left(r / w_{0}\right)^{m} G$.

Typical intensity and phase distributions for the optical quark are shown in Fig. 2. Here a distinct phase step $\pi$ along the corresponding rays, including the axis $r=0$, draws attention.

\subsection{Angular spectrum of the simplest optical quarks}

The propagation properties of the optical quarks are defined by their angular spectral functions

$$
\Psi\left(k_{p}, \phi\right)=\frac{k}{2 \pi} \int_{0}^{\infty} r d r \int_{0}^{2 \pi} d \varphi \Psi(r, \varphi) e^{-i k_{p} r \cos (\varphi-\phi)},
$$

where $k_{p}$ and $\phi$ are the radial and azimuthal coordinates in the $\mathbf{k}$ space, while $r$ and $\varphi$ are the polar coordinates.

At first we will analyse the angular spectrum of the optical quark with the BG envelope at the initial plane $z=0$, which is represented as

$$
Q_{e v}^{+, 1}=\cos \frac{\varphi}{2} e^{i \frac{\varphi}{2}} J_{1}(K r) e^{-r^{2} / w_{0}^{2}} .
$$

Since $\cos \frac{\varphi}{2} e^{i \frac{\varphi}{2}}=\frac{e^{i \varphi}+1}{2}$, the spectral integral given by Eq. (12) may be represented as a sum of two functions: 


$$
\Psi\left(k_{p}, \phi\right)=\Psi_{1}\left(k_{p}, \phi\right)+\Psi_{2}\left(k_{p}, \phi\right),
$$

The form of the $\Psi_{1,2}$ functions is defined by the $K$ parameter. If $K$ is real, we obtain

$$
\Psi_{1}\left(k_{p}, \phi\right)=-i z_{0} e^{i \phi} \exp \left\{-\frac{\left(K^{2}+k_{p}^{2}\right) w_{0}^{2}}{4}\right\} I_{1}\left(\frac{k_{p} K w_{0}^{2}}{2}\right),
$$

where $I_{m}(x)$ is the modified Bessel function of the first kind and the $m$ th order.

For the imaginary $K$ parameters one finds

$$
\Psi_{1}\left(k_{p}, \phi\right)=z_{0} e^{i \phi} \exp \left\{\frac{\left(K^{2}-k_{p}^{2}\right) w_{0}^{2}}{4}\right\} J_{1}\left(\frac{k_{p} K w_{0}^{2}}{2}\right),
$$

because of the property $I_{n}(i x)=i^{n} J_{n}(x)$, with $J_{m}(x)$ being the Bessel function of the first kind and the $m$ th order.

The second integral is

$$
\Psi_{2}\left(k_{p}\right)=z_{0} \frac{k_{p} w_{0}}{2} \sum_{m=0}^{\infty} \frac{\Gamma(m+3 / 2)}{(m !)^{2}}\left(-\frac{k_{p-}^{2} w_{0}^{2}}{4}\right)^{m}{ }_{2} F_{1}\left(-m,-(m+1) ; 1 ; \pm \frac{K^{2}}{k_{p}^{2}}\right)
$$

where the signs ' + ' and '-' refer respectively to real and imaginary $K$ parameters, ${ }_{2} F_{1}$ stands for the confluent hypergeometric function, and $\Gamma(x)$ the gamma-function.
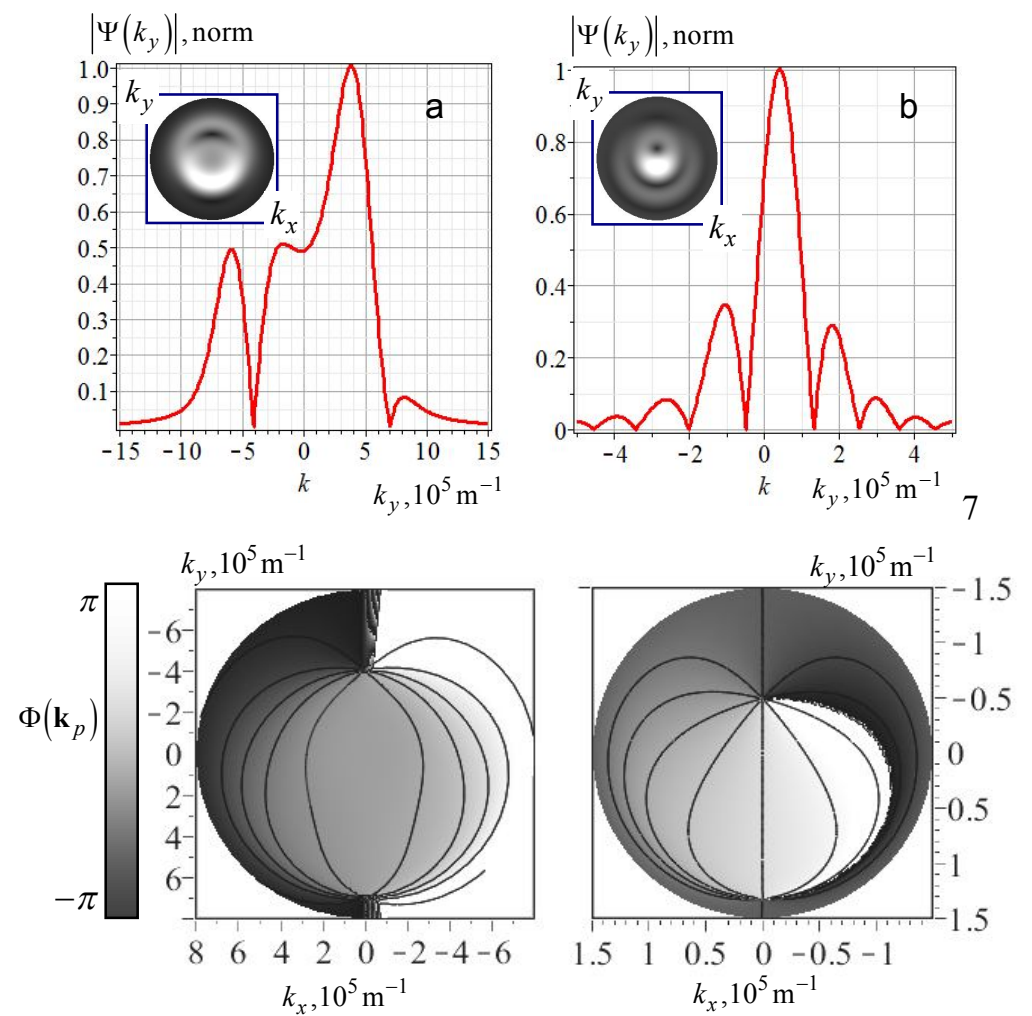

Fig. 3. Amplitude $\left|\Psi\left(k_{p}, \phi\right)\right|$ (a, b) and phase $\Phi\left(\mathbf{k}_{p}\right)=\arg \left(\Psi\left(k_{p}, \phi\right)\right)$ (c, d) for the optical quark $Q_{e v}^{+, 1}$ with the BG envelope: (a, c) $K=i 5 \times 10^{5} \mathrm{~m}^{-1}, w_{0}=10^{-5} \mathrm{~m}$ and (b,d) $K=5 \times 10^{5} \mathrm{~m}^{-1}, w_{0}=10^{-5} \mathrm{~m}$. 
Typical amplitude and phase distributions for the optical quark with the BG envelope are shown in Fig. 3 for both the real and imaginary $K$ parameters. First of all, these are complex angular spectra with a series of asymmetrically positioned main directions of the wave propagation. Second, there are topological dipoles (i.e., two oppositely charged phase screw singularities) in the $\mathbf{k}$-space phase distributions.

In a similar manner, one can construct the angular spectrum for the optical quarks with the simplest LG envelope at the $z=0$ plane:

$$
\Psi(r, \varphi)=\cos \frac{\varphi}{2} e^{i \frac{\varphi}{2}}\left(\frac{r}{w_{0}}\right) e^{-r^{2} / w_{0}^{2}} .
$$

Now we obtain two spectrum functions in Eq. (11) in the form

$$
\begin{gathered}
\Psi_{1}\left(k_{p}\right)=\frac{z_{0} \sqrt{\pi}}{2}\left[\left(1-\frac{k_{p}^{2} w_{0}^{2}}{4}\right) I_{0}\left(\frac{k_{p}^{2} w_{0}^{2}}{8}\right)+\frac{k_{p}^{2} w_{0}^{2}}{4} I_{1}\left(\frac{k_{p}^{2} w_{0}^{2}}{8}\right)\right] e^{-\frac{k_{p}^{2} w_{0}^{2}}{8}}, \\
\Psi_{2}\left(k_{p}, \phi\right)=\frac{k_{p} w_{0} z_{0} e^{i \phi}}{2} e^{-\frac{k_{p}^{2} w_{0}^{2}}{4}}
\end{gathered}
$$

Typical features of the angular spectrum for the optical quark with the LG envelope are shown in Fig. 4. The same as in case of the quark with the BG envelope, we observe a complex angular spectrum, with the topological dipole present. Availability of energy flux along the axis $k_{p}=0$ points to a transformation of structure of the quark along the beam axis. Besides, the topological dipole in the spectral function presupposes dislocation reactions in the wave structure while the beam is propagating. The above features are, by all appearances, inherent in either of the standard types of quark envelope in the free space.
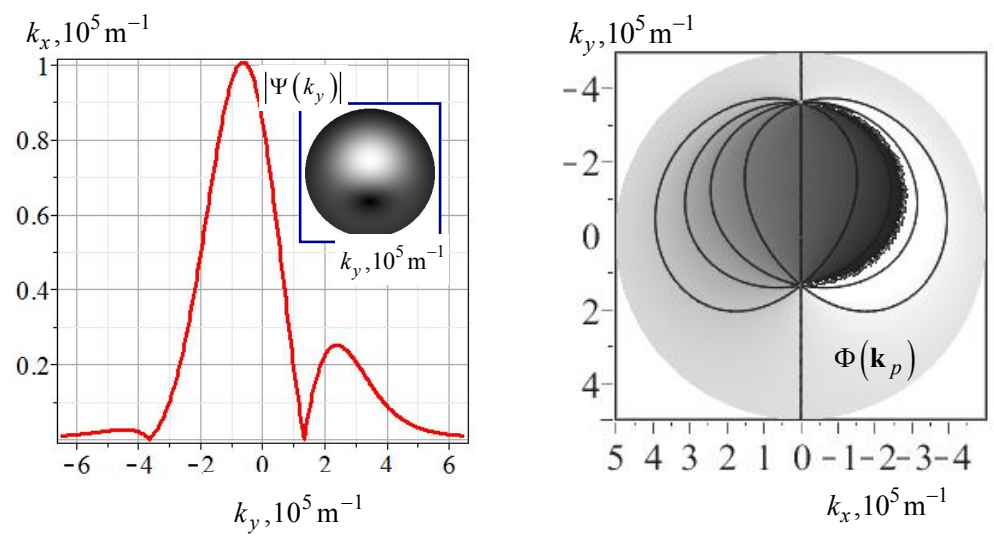

Fig. 4. Amplitude $\left(\left|\Psi\left(\mathbf{k}_{p}\right)\right|\right)$ and phase $\left(\Phi\left(\mathbf{k}_{p}\right)\right)$ distributions in angular spectrum of the optical quark $Q_{e v}^{+, 1}$ with the LG envelope.

\subsection{Free-space propagation of the optical quarks}

The detailed propagation process for the paraxial beams can be described in the framework of diffraction integral: 


$$
\Psi(r, \varphi, z)=\frac{C}{z} \int_{0}^{\infty} d r^{\prime} \int_{0}^{2 \pi} e^{-i k \sqrt{\left(x-x^{\prime}\right)^{2}+\left(y-y^{\prime}\right)^{2}+z^{2}}} \Psi\left(r, \varphi^{\prime}\right) d \varphi^{\prime}
$$

where $C$ is a constant. For the optical quark $Q_{e v}^{+, m}$ we have

$$
\Psi(r, \varphi, z)=\frac{C}{z} \int_{0}^{\infty} d r^{\prime} \int_{0}^{2 \pi} e^{-i k \sqrt{\left(x-x^{\prime}\right)^{2}+\left(y-y^{\prime}\right)^{2}+z^{2}}}\left\{\cos \left(m+\frac{1}{2}\right) \varphi^{\prime} e^{i\left(m+\frac{1}{2}\right) \varphi^{\prime}} r^{m} e^{-\frac{r^{\prime 2}}{w_{0}^{2}}}\right\} d \varphi^{\prime} .
$$

Therefore in the paraxial approximation for the wave zone we obtain

$$
\begin{aligned}
Q_{e v}^{+, m}(r, \varphi, z) & =\frac{C e^{-i k z-\frac{i k r^{2}}{2 z}}}{2 z} \int_{0}^{\infty} r^{\prime 2} d r^{\prime} e^{-\frac{i k r^{\prime 2}}{2 z}} e^{-\frac{r^{\prime 2}}{w_{0}^{2}} \int_{0}^{2 \pi}\left(1+e^{i m \varphi^{\prime}}\right) e^{i \frac{k r r^{\prime}}{z} \cos \left(\varphi-\varphi^{\prime}\right)} d \varphi^{\prime},} \\
& =\frac{C e^{-i k z}}{2}\left(\Psi_{m, 1}+\Psi_{m, 2}\right)
\end{aligned}
$$

with

$$
\begin{gathered}
\Psi_{1, m}=\frac{\Gamma\left(\frac{m+1}{2}\right) z^{\frac{m}{2}+1} e^{\frac{k z_{0}}{4\left(z+i z_{0}\right)} r^{2}} e^{-i k \frac{r^{2}}{2\left(z+i z_{0}\right)}} \times}{k^{2} r^{2}\left(z+i z_{0}\right)^{\frac{m}{2}}} \times \\
\times\left\{m M_{\frac{m}{2}, \frac{1}{2}}\left(\frac{k z_{0}}{2 z\left(z+i z_{0}\right)} r^{2}\right)-(2+m) M_{\frac{2+m}{2}}\left(\frac{k z_{0}}{2 z\left(z+i z_{0}\right)} r^{2}\right)\right\} \\
\Psi_{m, 2}=\left(\frac{z_{0}}{z+i z_{0}}\right)^{m} \frac{w_{0}^{2}}{z+i z_{0}}\left(\frac{r}{w_{0}}\right)^{m} e^{i m \varphi} \mathrm{e}^{-i k \frac{r^{2}}{2\left(z+i z_{0}\right)},}
\end{gathered}
$$

and $M_{\mu, v}(x)$ denoting the Whittaker function. For the simplest case of $m=1$ we find

$$
\begin{aligned}
\Psi & =\frac{\sqrt{z \pi} w_{0}^{2} e^{\frac{k z_{0}}{4 z\left(z+i z_{0}\right)} r^{2}}}{4\left(z+i z_{0}\right)^{\frac{3}{2}}} \times \\
& \left\{\left(1-\frac{k z_{0}}{2 z\left(z+i z_{0}\right)} r^{2}\right) I_{0}\left(\frac{k z_{0}}{4 z\left(z+i z_{0}\right)} r^{2}\right)+\frac{k z_{0}}{2 z\left(z+i z_{0}\right)} r^{2} I_{1}\left(\frac{k z_{0}}{4 z\left(z+i z_{0}\right)} r^{2}\right)\right\} e^{-i k \frac{r^{2}}{2\left(z+i z_{0}\right)}} .
\end{aligned}
$$

The wavefront transformations in the wave zone for the optical quarks with the simplest LG envelope are depicted in Fig. 5. A slight shift of the observation plane along the beam axis is accompanied by nucleating a chain of singly charged optical vortices with the opposite topological charges, which appear with increasing length. The field structure is simplified in the far-field zone (see Fig. 6). The vortex chains are extruded at infinity and the distance between them increases, so that only one singly charged optical vortex is observed near the beam axis.

Thus, the inner structure of the optical quark with a continuous phase distribution in the cross section of the beam is such that, whatever the standard envelope of the wave construction, it is inevitably accompanied by destruction of the quark. However, there are many other non-standard 
forms of the beam envelopes for the paraxial beams, which might nevertheless provide structural stability of the optical quarks. One of them is discrete optical vortices $[16,17]$.
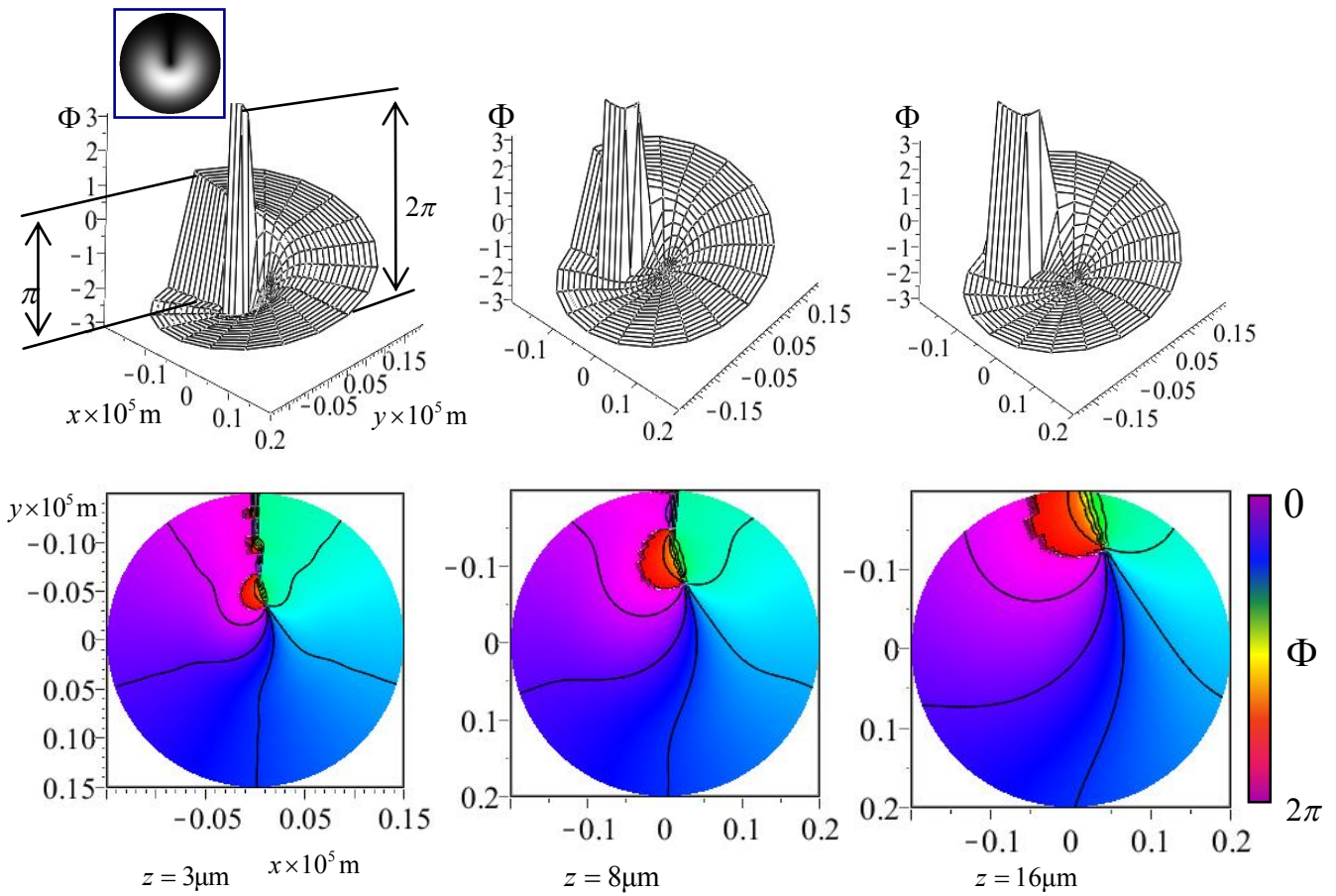

Fig. 5. Evolution of wavefront $\Phi(r, \varphi, z)$ for the optical quark $Q_{e v}^{+, 1}$ with $w_{0}=20 \mu \mathrm{m}$ along $z$ direction in the wave zone.
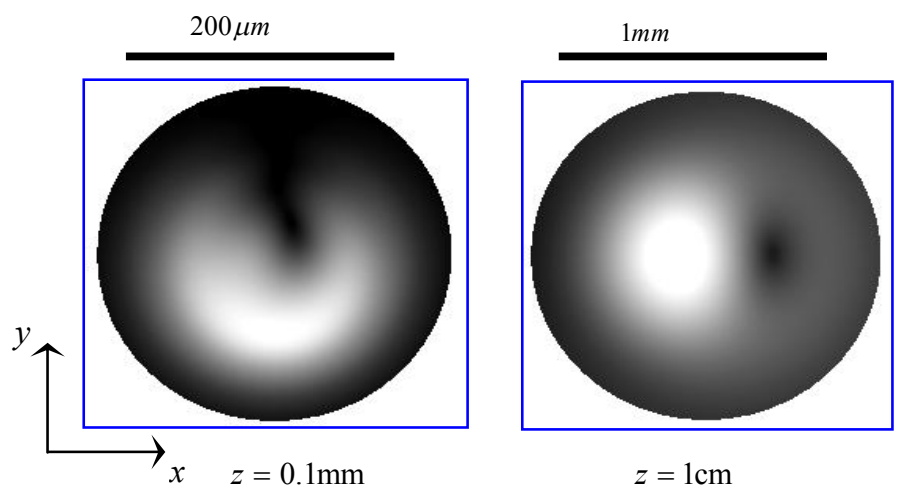

Fig. 6. Intensity distributions for the far-field zone.

\section{Discrete optical quarks}

A new type of screw wavefront singularities is given by discrete optical vortices shaped in the symmetrical beam arrays [16-19]. The beam array can be constructed in such a way that the field amplitude near the axis vanishes, thus essentially weakening process of destruction of the optical quark. Besides, variations of the array's parameters permit it to be transformed into a so-called spiral beam [17], a structurally stable wave construction of which shape is recovered up to the scale and rotation when propagating. 
In our further consideration we will hold to the approach suggested in the study [19]. Let us consider a symmetric array of the $\mathrm{G}$ beams shown in Fig. 7. Each of the $\mathrm{G}$ beams is located at the vertices of the regular polygon (with $x_{n}^{\prime}, y_{n}^{\prime}, z$ being the local coordinates) at the distance $r_{0}$ from the origin of the global reference frame. The $z$ axis of each local beam is tilted by the angle $\alpha$ in the $\left(y_{n}^{\prime}, z\right)$ plane, where $x_{n}=x_{n}^{\prime}, y_{n}, z_{n}$ are the coordinates.
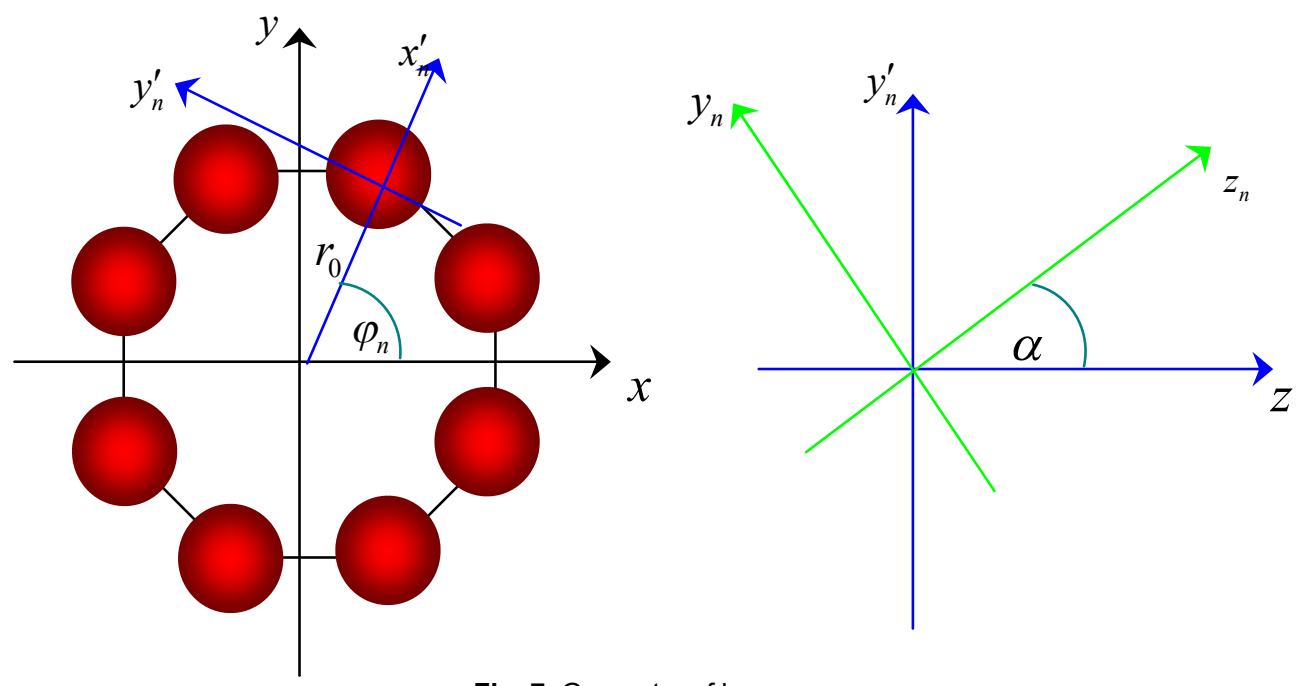

Fig. 7. Geometry of beam array.

The beam coordinates are linked to the coordinates in the global referent frame $(x, y, z)$ :

$$
x_{n}=r \cos \left(\varphi+\varphi_{n}\right)+r_{0}, y_{n}=r \sin \left(\varphi+\varphi_{n}\right)-\alpha z, z_{n}=\alpha r \sin \left(\varphi+\varphi_{n}\right)+z,
$$

where we have used the paraxial approximation ( $\sin \alpha \approx \alpha), r$ and $\varphi$ are the polar coordinates of the global referent frame, $\varphi_{n}=n \frac{2 \pi}{N}, n=0,1,2, \ldots, N-1$ is the site of the local beam, and $N$ the number of local beams in the array. Then the local phase $\Delta_{n, q}=\varphi_{n} q$ and the amplitude $f_{n, q}$ (with $q$ being an arbitrary real number) are ascribed to each beam. Thus, the scalar field of the beam array may be obtained in the following form:

$$
\Psi_{q}=\frac{1}{N} \sum_{n=0}^{N-1} f_{n, q} e^{i \Delta_{n, q}} \frac{\exp \left\{-i k \frac{x_{n}^{2}+y_{n}^{2}}{2 Z_{n}}\right\}}{Z_{n} /\left(i z_{0}\right)} e^{-i k z_{n}},
$$

with $Z_{n}=z_{n}+i z_{0}$.

When the number $q$ is integer and $f_{n, q}=1$, the total pass-by of the beam axis changes the beam phase by $2 \pi q$. The beam gets the optical vortex with the topological charge equal to $q$. However the phase for the local beams changes step by step rather than continuously. Such a 'strange' vortex beam has come to be called a discrete optical vortex [16]. The same situation occurs when $q=m / 2$, where $m$ is an odd number. However, here the phase changes step by step by the total value $\pi m n / N$, whereas the amplitude is $\cos \left(\frac{\pi m}{N} n\right)$ or $\sin \left(\frac{\pi m}{N} n\right)$. We call these 
structures as discrete optical quarks.

The simplest case of the discrete optical quark formed by the beam array, with all the axes of the local beams being parallel $(\alpha=0)$ to the global axis $z$ and $f_{n, m}=\cos \left(\frac{\pi m}{N} n\right), \Delta_{n, m}=\frac{\pi m}{N} n$, is shown in Fig. 8 in the initial plane $z=0$. In contrast to ideal phase patterns of the quark in Fig. 2, the phase step in this case has a non-uniform shape. Although the phase step remains to be $\pi$ far from the axis, the phase gradually reaches the value $\pi$ near the array axis. Besides, we do not deal hear with the spiral beam and its structure must therefore change as the beam propagates. Indeed, Fig. 9 demonstrates that the intensity distribution for the optical quark with $q=5 / 2$ evolves very quickly along the array axis, so that the beam gets a rather regular tracery of singly charged optical vortices in the far-field zone.
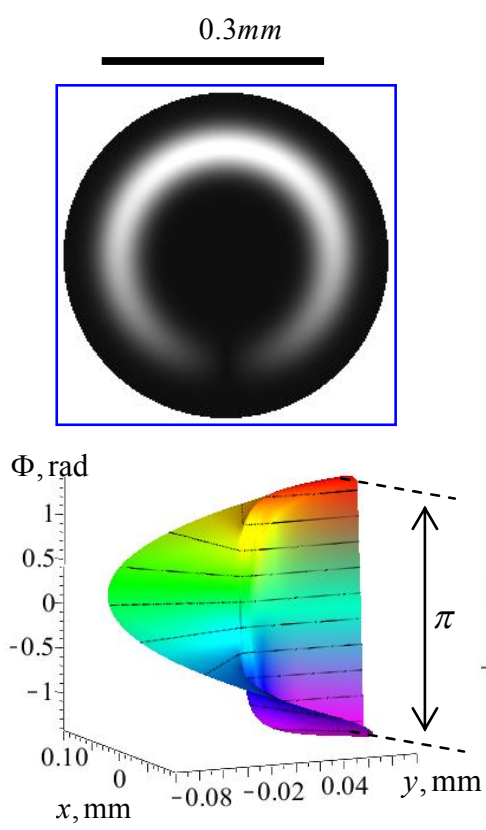

$q=1 / 2$
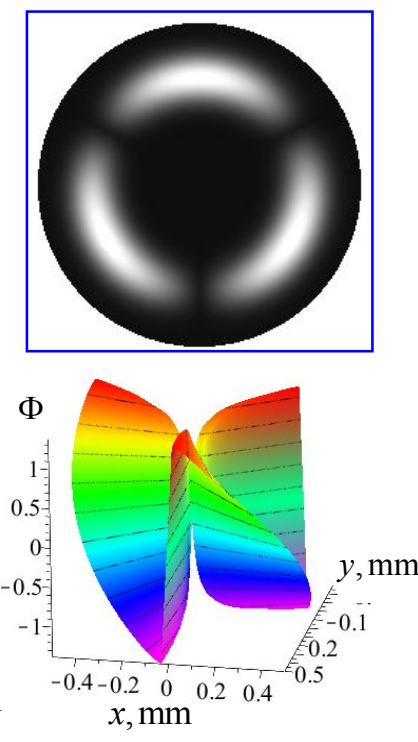

$q=3 / 2$
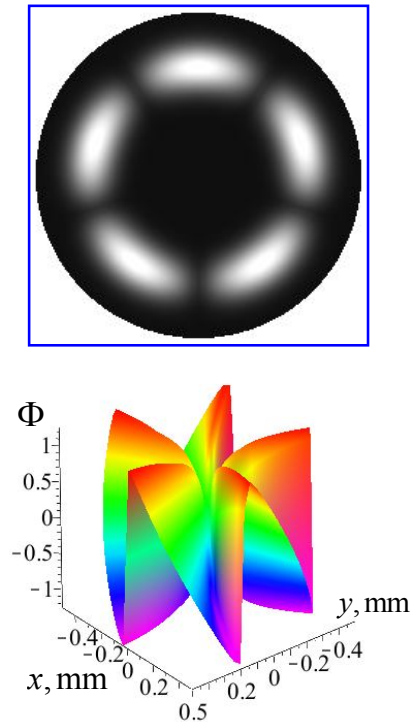

$q=5 / 2$

Fig. 8. Intensity $(I)$ and phase ( $\Phi$ ) distributions for discrete optical quarks with $N=100$, $w_{0}=20 \mu \mathrm{m}, \alpha=0$ and $r_{0}=0.1 \mathrm{~mm}$ at $z=0$.

From the other hand, the beam array turns into the spiral beam under the condition $r_{0}=\alpha z_{0}$, at least for the integer $q$ values [17]. This means that the tilt of the local beams is compensated by the diffraction process so that the field is recovered up to scaling and rotation. It is just the case shown in Fig. 10. Here we observe a cylindrical phase plate with $\Phi=0$ in the vicinity of the array axis. The corresponding $\pi$ steps are shifted though they retain a regular form of the step (in contrast to what is shown in Fig. 8). The spatial evolution of the optical quark displayed in Fig. 11 represents a destruction process. The shape of the wavefront is gradually deformed, the spiral beam loses its self-recovering properties in the case of fractional $q$ indices, and the discrete optical quark is broken down. At the same time, the sum of the even and odd quarks remains to be an invariant structure. 


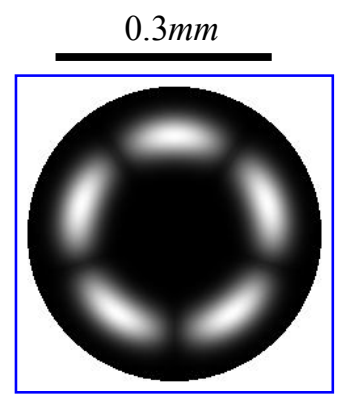

$z=20 \mu \mathrm{m}$

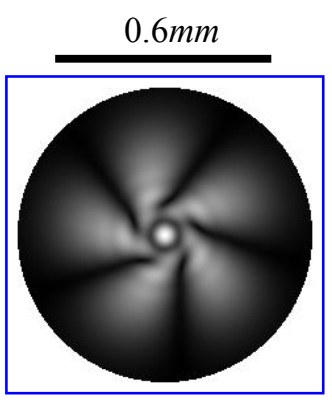

$z=1 \mathrm{~cm}$

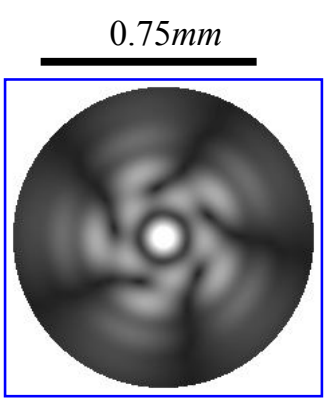

$2 \mathrm{~cm}$

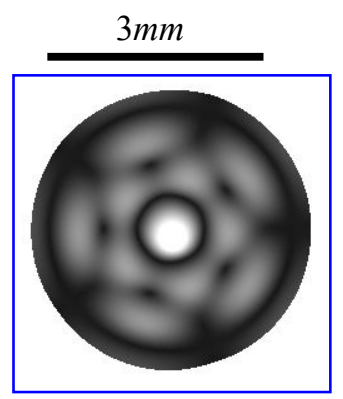

$z=30 \mathrm{~cm}$

Fig.9. Spatial evolution of intensity distribution for the discrete optical quark with $q=5 / 2$, $N=100, w_{0}=20 \mu \mathrm{m}, \alpha=0$ and $r_{0}=0.1 \mathrm{~mm}$
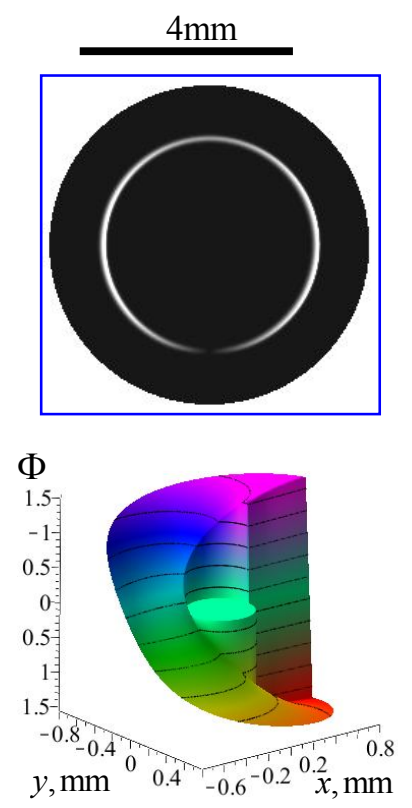

$$
q=1 / 2
$$
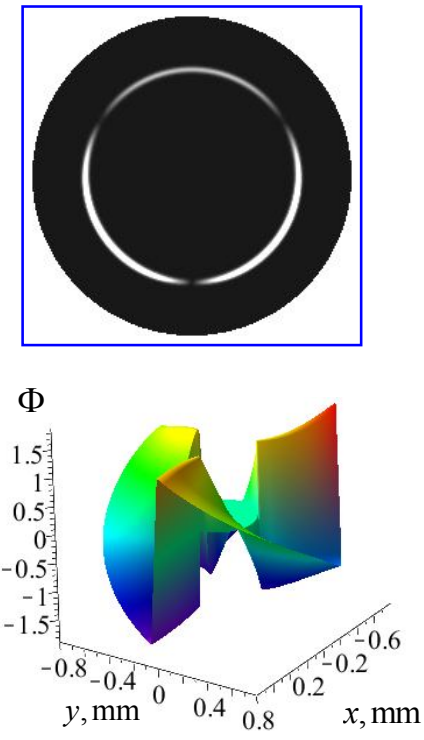

$q=3 / 2$

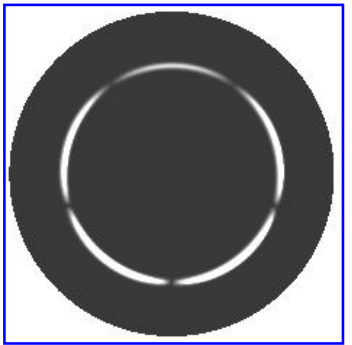

$\Phi$

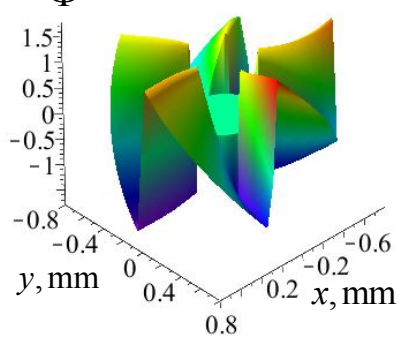

$q=5 / 2$

Fig. 10. Discrete quarks in the spiral beam with $N=100, r_{0}=4 \mathrm{~mm}, \alpha=0.0008$ and $w_{0}=20 \mu \mathrm{m}$ at $z=0$.

\section{Conclusions}

We have considered a new type of singular beams with the fractional topological charges, which possess rather unique properties and have been called as the optical quarks. The corresponding topological charges are half of integer order. There are four types of the optical quarks (even and odd ones, with the opposite signs of their topological charges). The sums or the differences of the even and odd quarks form respectively standard vortex or non-vortex beams, with the integerorder topological charges. All the quarks in the same beam annihilate. The analysis of angular spectra of the quarks testifies a presence of spectral components directed along the beam axis. This implies that the wave state of the quark is structurally unstable when the beam is propagating. We have analysed the propagation properties of the optical beams with different types of standard en- 
velopes, including a symmetric beam array in both arbitrary and self-recovering (spiral-beam) states. We have revealed that the spiral beams with the half-order indices lose their self-recovering properties, thus causing a break down of the quark.
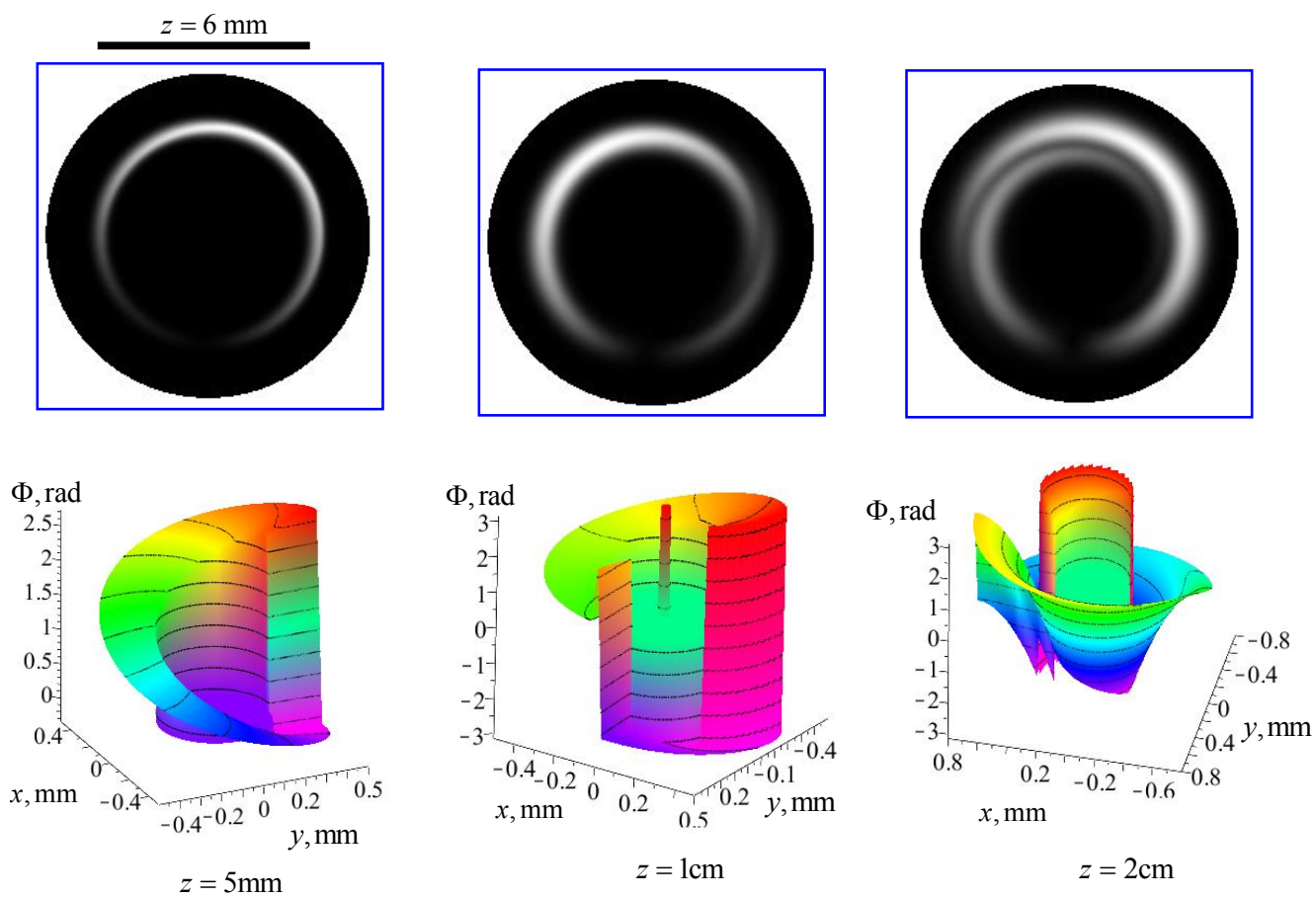

Fig. 11. Spatial evolution of optical quark in the spiral beam $(q=1 / 2)$.

Destruction of the optical quarks in the free space puts definite restrictions onto the beam structure and the properties of optical medium capable to preserve the optical quarks. To our opinion, a structurally stable optical quark must represent, first of all, a vector field whose circularly polarised components have topological charges of half-integer orders differing by a unit value. Besides, the medium has to reveal birefringent properties, with the principal directions of the relevant tensor forming a field with a fractional topological index.

\section{References}

1. Basisty I, Soskin M and Vasnetsov M, 1995. Optical wavefront dislocations and their properties. Opt. Commun. 119: 604-612.

2. Basisty I, Pas'ko V, Slyusar V, Soskin M and Vasnetsov M, 2004. Synthesis and analysis of optical vortices with fractional topological charges. J. Opt. A: Pure Appl. Opt. 6: S166-S169.

3. Berry M V, 2004. Optical vortices evolving from helicoidal integer and fractional phase steps. J. Opt. A. 6: 259-269.

4. Leach J, Yao E and Padgett M J, 2004. Observation of the vortex structure of a non-integer vortex beam. New J. Phys. 6: 71 .

5. Garcia J Gutierrez-Vega, 2009. Diffraction of plane waves by finite-radius spiral phase plates of integer and fractional topological charge. J. Opt. Soc. Amer. A. 26: 794-803.

6. Gutiérrez-Vega J C and López-Mariscal C, 2008. Nondiffracting vortex beams with continuous orbital angular momentum order dependence. J. Opt. A: Pure Appl. Opt. 10: 015009. 
7. Mitri F G, 2011. Vector wave analysis of an electromagnetic high-order Bessel vortex beam of fractional type $\alpha$. Opt. Lett. 36: 606-608.

8. Guti'errez-Vega J C, 2007. Fractionalization of optical beams: I. Planar analysis. Opt. Lett. 32: $1521-1523$.

9. Guti'errez-Vega J C, 2007. Fractionalization of optical beams: II. Elegant Laguerre-Gaussian modes. Opt. Express. 15: 6300-5313.

10. Oemrawsingh S S R, Ma X, Voigt D, Aiello A, Eliel E R, G W 't Hooft and Woerdman J P, 2005. Experimental demonstration of fractional orbital angular momentum entanglement of two photons. Phys. Rev. Lett. 95: 240501.

11. Gotte J, Franke-Arnold S, Zambrini R and Barnett S M, 2007. Quantum formulation of fractional orbital angular momentum. J. Mod. Opt. 54: 1723-1738.

12. Fadeyeva T, Alexeyev C, Rubass A and Volyar A, 2012. Vector erf-Gaussian beams: fractional optical vortices and asymmetric TE and TM modes. Opt. Lett. 37: 1397-1399.

13. Nugrowati A M, Stam W G and Woerdman J P, 2012. Position measurement of non-integer OAM beams with structurally invariant propagation. Opt. Express. 20: 27429-27440.

14. Berry M V, Chambers R G, Large M D, Upstill C and Walmsley J C, 1980. Wavefront dislocations in the Aharonov-Bohm effect and its water wave analogue. Eur. J. Phys. 1: 154-162.

15. Wong S S M. Introductory nuclear physics, $2^{\text {nd }}$ edition, Morlenbach: Wiley Interscience Publication, (1998).

16. Alexeyev C N, Volyar A V and Yavorsky M A, 2009. Linear azimuthons in circular fiber arrays and optical angular momentum of discrete optical vortices. Phys. Rev. A. 80: 063821.

17. Izdebskaya Ya, Shvedov V and Volyar A, 2008. Symmetric array of off-axis singular beams: spiral beams and their critical points. J. Opt. Soc. Amer. A. 25: 171-181.

18. Izdebskaya Ya, Fadeyeva T, Shvedov V and Volyar A, 2006. Vortex-bearing array of singular beams with very high orbital angular momentum. Opt. Lett. 31: 2523- 2525.

19. Volyar A, Shvedov V, Izdebskaya Ya, Fadeyeva T and Rubass A, 2006. Structure and orbital angular momentum of singular array of Gaussian beams. Ukr. J. Phys. Opt. 7: 79-88.

Volyar Alexander V., 2013. Do optical quarks exist in the free space? A scalar treatment. Ukr.J.Phys.Opt. 14: $31-43$.

Анотація. Ми розглянули новий тип сингулярних пучків, названий оптичними кварками. Вони мають дробовий топологічний заряд, рівний половині цілого числа і володіють унікальними властивостями. Існуе чотири типи оптичних кварків, парні і непарні, які мають протилежний знак топологічного заряду. Сума або різниия парних і непарних кварків формуе стандартні вихрові або невихрові пучки з иілим топологічним зарядом. Всі кварки у одному і тому ж промені анісілюють. Аналіз кутових спектрів оптичних кварків засвідчуе, що кварки являють собою структурно нестабільну форму поля при поширенні у вільному просторі. Ми проаналізували їх властивості при поширенні різних типів пакетів, включаючи симетричний масив променів з дискретними оптичними кварками. В роботі обговорюються властивості структурно стабільних форм кварків і середовища здатні підтримувати оптичні кварки. 